\title{
Granulicatella elegans
}

National Cancer Institute

\section{Source}

National Cancer Institute. Granulicatella elegans. NCI Thesaurus. Code C111204.

A species of facultative anaerobic, gram-positive, cocci shaped bacteria assigned to the phylum Firmicutes. This species is non-motile, non-spore forming and catalase and oxidase negative. G. elegans is commensal, being found in normal human oral flora, but may be associated with infective endocarditis. 